\title{
Effective Beamforming Technique for Carrier Aggregation of Millimeter Wave
}

\author{
Tae-Kyoung Kim¹)
}

\begin{abstract}
This paper proposes an effective beamforming technique for carrier aggregation of millimeter wave. Millimeter wave exploits a high center frequency that results in a large path loss. To overcome this large path loss, analog beamforming was introduced using a phase shifter. Analog beamforming can be extended to the carrier aggregation system that is composed of a primary carrier component (PCC) and secondary carrier component (SCC). Generally, each carrier component has its radio frequency (RF) chain so that beamforming is independently performed. However, the expense of a millimeter RF chain is extremely high. Thus, it limits the number of RF chains in the terminal. To address this problem, this paper proposes an effective beam selection method that involves sharing of the RF chain by the carrier component. Performance of the PCC should be guaranteed because the PCC conveys an important system parameter to the terminal. The beam selection problem is first designed considering this constraint, followed by proposing an effective solution to the problem. This study investigates the effectiveness of the proposed method in terms of throughput and complexity. Simulations show that the proposed solution provides higher throughput than the conventional PCC-based method while the throughput of PCC is guaranteed.
\end{abstract}

Keywords : Beamforming, Carrier Aggregation, Millimeter Wave, Primary Carrier Component, Throughput

\section{Introduction}

Millimeter wave (mmWave) is a popular wireless communication technology because of its high band spectrum[1-3]. Third Generation Partnership Project (3GPP) has adopted the mmWave band at the Fifth Generation New Radio (5G NR) standard using $28 \mathrm{GHz}$ and $39 \mathrm{GHz}[4-6]$. Short wavelength in mmWave allows large amount of antenna elements. As a result, the mmWave system is easy to apply to the multi-input multi-output (MIMO) technique. Due to the large-scale MIMO technique, the mmWave system can utilize analog beamforming. Analog beamforming can be controlled by a phase shifter implemented in the radio frequency (RF) [7-9]. From the analog beamforming, the mmWave system can overcome a significant path loss,

Received(November 15, 2018), Review Result(1st: November 30, 2018, 2nd: January 10, 2019), Accepted(March 10, 2019)

1) (Professor) 58554 Department of Electronics, Information and Communications Engineering, Mokpo National University, Muan, Jeonnam, South Korea

email: tk415kim@gmail.com 
which is usually a problem in the mmWave channels.

Carrier aggregation also enables the exploitation of more spectrum band by means of aggregating multiple small bands, which is called a component carrier (CC)[10][11]. Information of each CC can be transmitted from the source and is collected at the destination. This makes the extension of scalable bandwidth possible. Component carriers can be classified as primary CCs (PCCs) and secondary CCs (SCCs)[12][13]. PCC is initially used to establish a connection between the base station (BS) and user. After the connection, important system parameters are exchanged through the PCC channels. SCCs are typically used to improve the throughput and are favored for burst data transmission.

Generally, carrier aggregation is easily extended to a beamforming system[14][15]. In conventional extension, each $\mathrm{CC}$ has its separate RF chain (Fig. 1(a)). As a result, the number of RF chains is the same as that of CCs. By using a separate RF chain, each CC can independently control a beam-steering antenna. However, with the mmWave system, implementation of multiple RF chains is difficult in a terminal because of their high cost and power consumption[16-21]. Hence, a smaller number of RF chains are used so that adjacent CCs should be shared with an RF chain. Beam of adjacent CCs that share the same RF chain necessarily is steered together (Fig. 1(b)). Although, this RF sharing problem is remarkable at the mmWave system, but there is no criterion of deciding the direction of analog beamforming among the CCs yet. When the PCCs and SCCs co-exist, the beamforming method becomes more complicated because their roles are different.

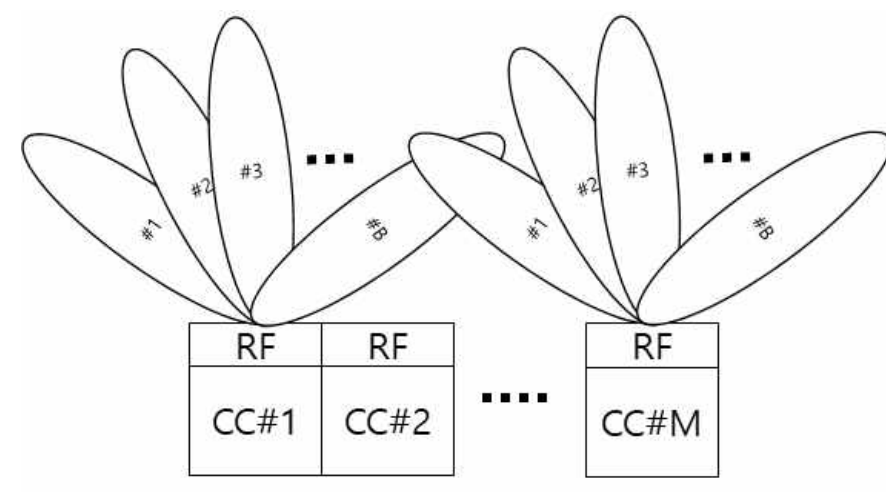

(a)

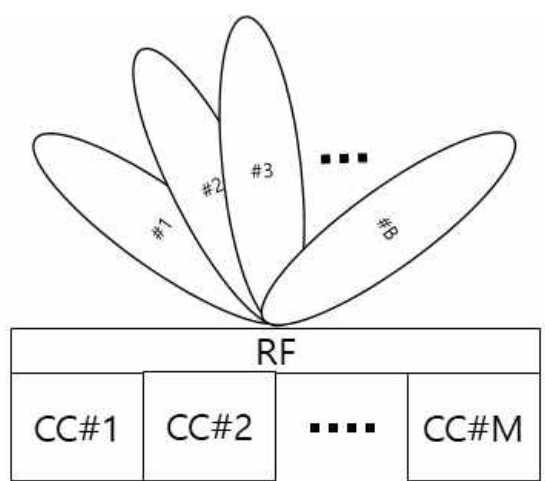

(b)

[Fig. 1] Carrier Aggregation System with Analog Beamforming (a) Conventional System

(b) mmWave System 
This study investigates carrier aggregation of analog beamforming. To this end, the beam selection problem is formulated when the PCCs and SCCs co-exist. The formulation maximizes the system throughput while guaranteeing the performance of the PCC. To solve this problem, an effective method is proposed that first investigates the performance of the PCC. When the performance threshold of the PCC is not met, analog beam is selected based on the performance of the PCC. On the other hand, when the performance of the PCC is guaranteed, the proposed method maximizes the system throughput. In addition to the throughput, the complexity of the proposed method is investigated using various methods. Simulation results show that the throughput in case of the proposed method is higher than the conventional PCC-based method. As the SNR increases, the proposed method is close to the TPUT-based solution while its complexity is lower than that of the TPUT-based method.

\section{System model}

\subsection{Carrier Aggregation System with Analog Beamforming}

This study considers a carrier aggregation system in which a user has $\mathrm{M}$ number of CCs, including one PCC and (M-1) SCCs. For simplicity, the PCC and SCCs are defined as $C_{1}$ and $C_{n}, \mathrm{n}=2 \ldots, \mathrm{M}$, respectively. To reduce the space and cost, the PCC and SCCs are assumed to share the RF (Fig. 1(b)). Because all CCs share the RF, their direction is simultaneously changed. In contrast, in a conventional system at lower band, each CC has its own RF chain (Fig. 1(a)). Let $\Phi=\{1,2, \ldots, B\}$ denote the index set of the analog beam where $\mathrm{B}$ is the total number of analog beamforming (Fig. 1). Then, the source should choose one beam among the index set to communicate with the destination.

The detailed beamforming process is shown above (see Fig. 2). The first step is the beam training step, in which the beam source (BS) sends a beam training sequence to the user (destination) according to the beam index, n. Its direction is depicted in Fig. 1. Second is the measurement step. The associated user measures the signal strength whenever the beam training sequence is received. After that, the BS increases the beam index as $(n+1)$ and changes the beam direction. The BS performs this beam change until the number of beam index reaches B. Third is the beam selection step. In this step, the user selects the beam index by comparing the signal strength. Then, the user utilizes the selected beam index for data transmission. 


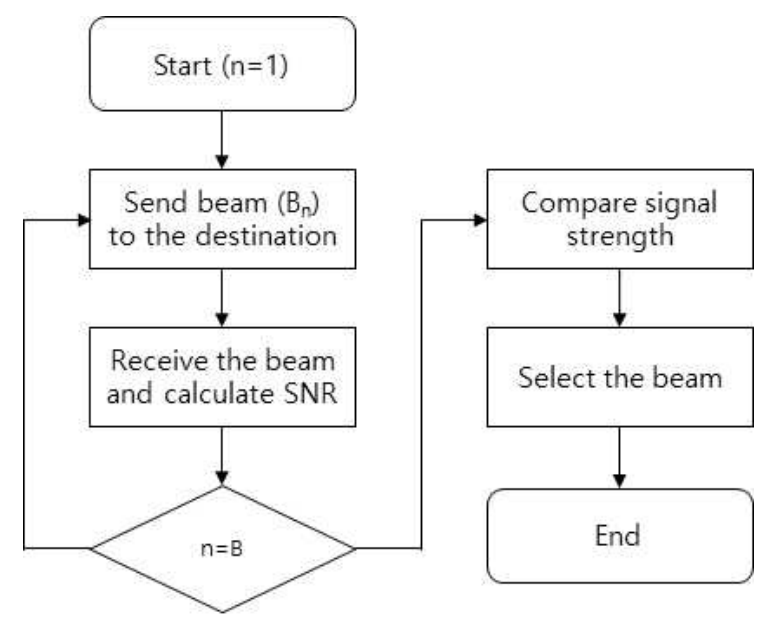

[Fig. 2] Flow Chart of Beam Selection

This study focuses on the beam selection criterion related to the third step. Especially, this study considers a carrier aggregation system with one RF. To address the issue of a carrier aggregation system with one $\mathrm{RF}$, the constrained problem is first formulated. Thereafter, the proposed method aimed to solve the problem is introduced in this paper.

\subsection{Problem Formulation}

The BS conveys system parameters to the user through the PCC. The system parameters should be perfectly decoded to prevent a connection failure. Thus, the minimum performance of the PCC should be guaranteed. From this condition, the problem is designed as:

$$
n=\arg _{\max } \sum_{b \in \Phi} \sum_{n=1}^{M} \log \left(1+S N R_{C_{n}}(b)\right), \text { s.t.SNR} R_{C_{1}}(b)>S N R_{t h}
$$

where $S N R_{C_{n}}(b)$ is the signal-to-noise ratio of the i-th CC $C_{n}$ and is the function of beam index $b$. This is because the channel is different whenever the beam is changed. In addition, the proposed system uses on RF so that the beam index has the same value for each CC $C_{n}$. The performance of PCC and minimum SNR are respectively defined as $S N R_{C_{n}}(b)$ and $S N R_{t h}$, in which the user can decode the system information. The summation term is the total throughput including the PCC and SCCs. The solution in (1) maximizes the total throughput, 
which is the role of the SCC while the performance of the PCC is guaranteed. In addition, the solution is difficult to obtain in a closed form because the beam index is a discrete set[22]. Therefore, in the next subsection, a heuristic solution is introduced. The heuristic solution is intuitive and provides the optimal solution of (1).

\subsection{Proposed Method}

This subsection describes the solution introduced in (1). The proposed method first checks the SNR of the PCC whenever the beam sequence is received, considering the importance of performance of the PCC to the system.. When the SNR of the PCC is below the threshold at a certain beam index, performance of the SCCs at this beam index is not calculated. When the SNR of the PCC is above the threshold, the proposed method calculates the total throughput as given in (1). From this condition, the complexity of the proposed method is considerably reduced at a low SNR, but the throughput is better than the conventional PCC-based method. After that, the proposed method finds the best beam index having the maximum throughput by comparing the total throughput as given in (1). The best beam index is the solution of problem described in (1). The detailed flow chart is presented in figure 3.

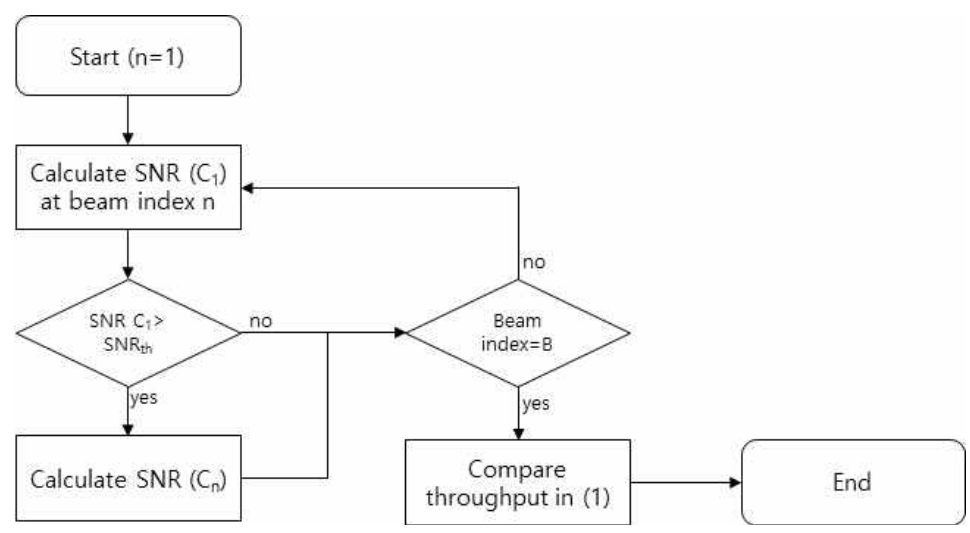

[Fig. 3] Flow Chart of the Proposed Method

\section{Simulation Results}

\subsection{Throughput}

We consider a carrier aggregation system with analog beamforming. This study assumes that 
each beam experiences an independent channel modelled by a Gaussian. The total number $\mathrm{M}$ of CCs is 8, which includes one PCC and 7 SCCS. The total number B of beam set is 14 . The minimum threshold is $0 \mathrm{~dB}$. This is because most system parameters are decoded by the QPSK symbol with a lower coding rate. Upon comparing the proposed method with the PCC-based and TPUT-based methods, it is observed that the PCC-based method uses only the performance of a PCC to select the beam index. This method is a simple but stable in the system. On the other hand, the TPUT-based method selects the beam index to maximize the total throughput but does not consider the performance of a PCC.

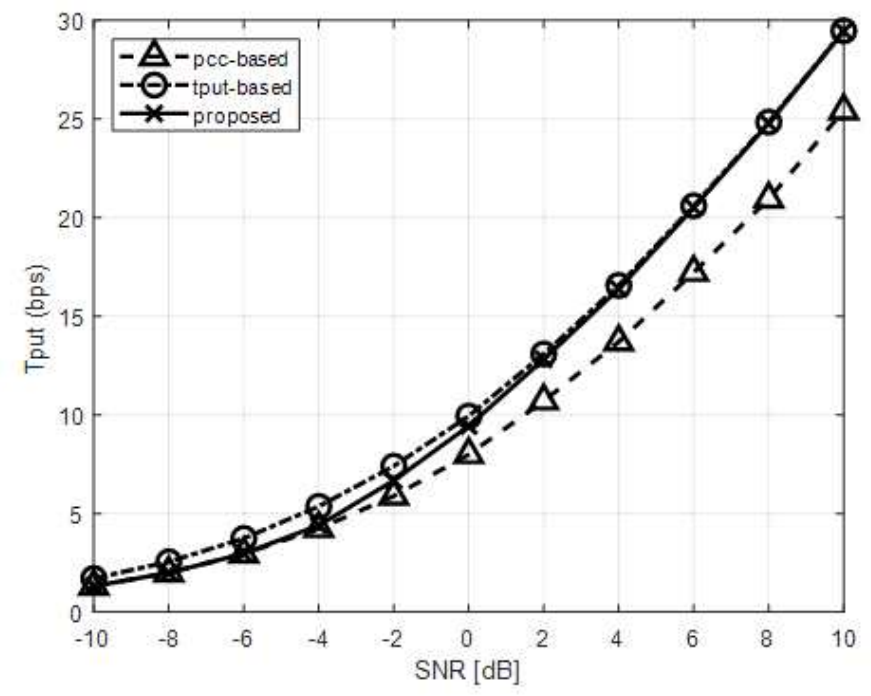

[Fig. 4] Total Throughput according to SNR when SNR_\{th $\}=0 \mathrm{~dB}$

Figure 4 shows the total throughput for different SNRs. At a low SNR, the throughput between the proposed and PCC methods is similar. This is because the performance of the PCC is difficult to satisfy. Thus, the proposed method selects the beam index on the basis of the PCC. However, as SNR is increased, the performance of the PCC is also improved. Thus, the performance of the proposed method is close to that of the TPUT-based method.

Figure 5 shows the throughput of the PCC according to different methods. The PCC-based method selects the favorable beam for the PCC so that its throughput is the highest. At a low SNR, the proposed method acts as the PCC-based method. Thus, its performance is similar to that of the PCC-based method. However, as SNR is increased, the throughput of the proposed method does not increase; whereas, the throughput of the PCC-based method reaches the threshold, which is the throughput at $0 \mathrm{~dB}$. Throughput of the proposed method increases after the cross-point between the throughput of the TPUT-based method and the threshold. From 
this point, the proposed method has the lowest throughput of PCC because it guarantees the minimum performance of the PCC. Note that the total throughput of the proposed method is similar to that of the TPUT-based method (Fig. 4).

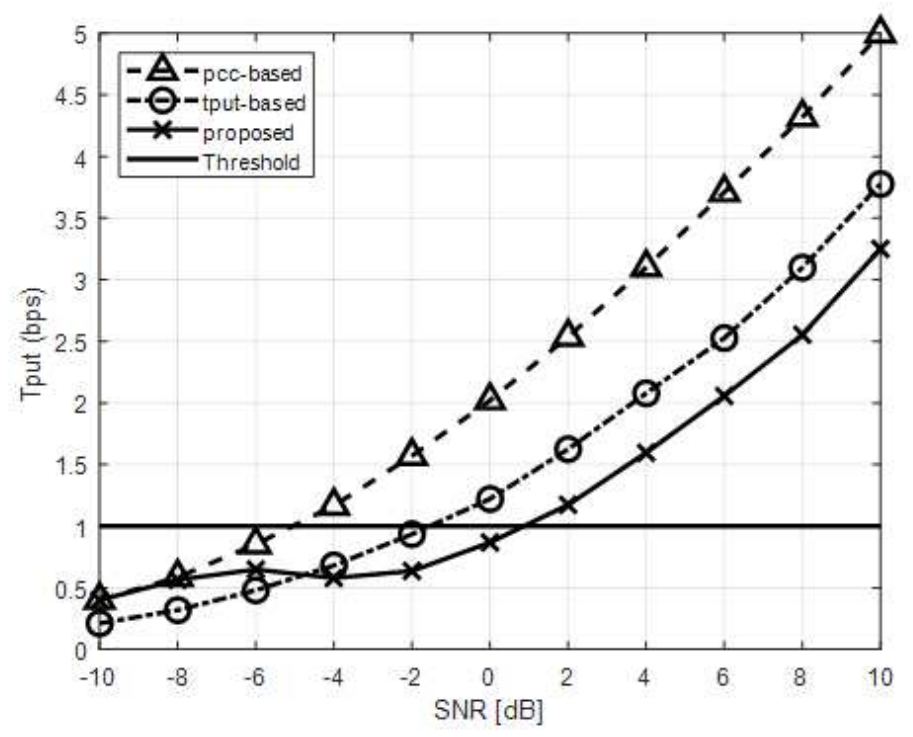

[Fig. 5] Throughput of PCC according to SNR when SNR_\{th $\}=0 \mathrm{~dB}$

\subsection{Complexity}

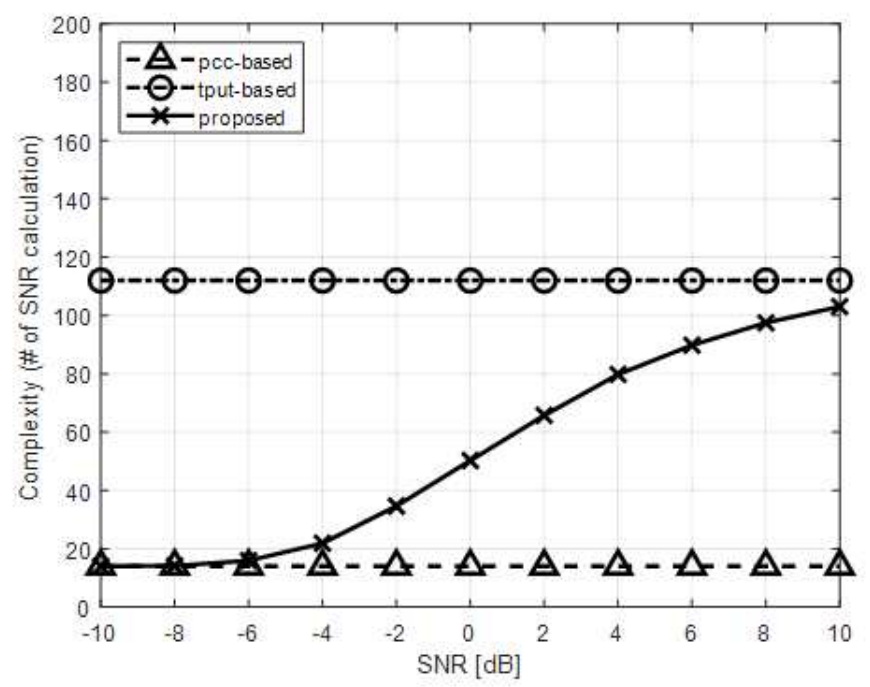

[Fig. 6] Complexity for Different Methods when $M=8$ and $B=14$. 
In this subsection, the complexities of various methods are compared (Fig. 6). For the comparison, the number of SNR calculations is investigated. Because the PCC-based method only compares the SNR of PCC, its complexity is the same as the number of beams. In contrast, the TPUT-based method considers all the cases same as $B^{*} \mathrm{M}$. The proposed method acts as the PCC-based method at a low SNR. Therefore, its complexity is similar to that of the PCC-based method. However, as the throughput of the PCC is guaranteed, the proposed method acts similar to the TPUT-based method. Because it investigates all combinations of beams and CCs, complexity of the proposed method is increased.

\section{Conclusions}

This paper proposed an effective beamforming technique for carrier aggregation of mmWave. In this study, the CCs shared the RF chain because of the limitation of cost and space. Thus, analog beamforming was applied to the same direction. To address this constraint, the beam selection problem is formulated to maximize the throughput while guaranteeing the performance of the PCC. The proposed method effectively finds the solution by comparing the SNR of PCC. Simulation results show that the proposed method improves the throughput over the conventional PCC-based method. This paper only considered the problem with one RF in the beamforming system, However, the number of RF could be increased by reducing its size. Thus, the proposed beam selection problem could be complicated with multiple RFs. The beamforming system with multiple RFs is the future work for this paper.

\section{References}

[1] T. Bai. R. W. Heath, Coverage and rate analysis for millimeter-wave celluar networks, IEEE Transactions on Wireless Communications, (2015), Vol.14, No.2, pp.1100-1114, DOI: 10.1109/TWC.2014.2364267

[2] W. Roh, J. Y. Seol, J. Park, B. Lee, J. Lee, Y. kim, J. Cho, K. Cheun, F. Aryanfar, Millimeter-wave beamforming as an enabling technology for $5 \mathrm{G}$ celluar communications: theoretical feasibility and prototype results, IEEE Communications Magazine, (2014), Vol.52, No.2, pp.106-113, DOI: 10.1109/MCOM.2014.6736750

[3] S. Hur, T. Kim, D. J. Love, J. V. Krogmeier, T. A. Thomas, A. Ghosh, Millimeter wave beamforming for wireless backhaul and access in small cell networks, IEEE Transactions on Communications, (2013), Vol.61, No.10, pp.4391-4403, DOI: 10.1109/TCOMM.2013.090513.120848 
[4] S. Parkvall, E. Dahlman, A. Furuskar, M. Frenne, NR: The new 5G radio access technology, IEEE Communications Standards Magazine, (2017), Vol.1, No.4, pp.24-30, DOI: 10.1109/MCOMSTD.2017.1700042

[5] J. G. Andrews, S. Buzzi, W. Choi, S. V. Hanly, A. Lozano, A. C. K. Soong, J. Zhang, What will be 5G be?, IEEE Journal on Selected Areas in Communications, (2014), Vol.32, No.6, pp.1065-1082, DOI: 10.1109/JSAC.2014.2328098

[6] NR - Physical channels and modulation, 3GPP TS 38.211 version 15.0.0 Release 15, ESTI, (2018)

[7] A. F. Molisch, V. V. Ratnam, S. Han, Z. Li, S. Nguyen, L. Li, K. Haneda, Hybrid beamforming for massive MIMO: A survey, IEEE Communications Magazine, (2017), Vol.55, No.9, pp.134-141, DOI: 10.1109/MCOM.2017.1600400

[8] S. Han, C.-L. I, Z. Xu, C. Rowell, Large-scale antenna systems with hybrid analog and digital beamforming for millimeter wave 5G, IEEE Communications Magazine, (2015), Vol.53, No.1, pp.186-194, DOI: 10.1109/MCOM.2015.7010533

[9] F. Sohrabi, W. Yu, Hybrid analog and digital beamforming for mmWave OFDM large-scale antenna arrays, IEEE Journal on Selected Areas in Communications, (2017), Vol.35, No.7, pp.1432-1443, DOI: 10.1109/JSAC.2017.2698958

[10] Z. Shen, A. Papasakellaiou, J. Montojo, D. Gerstenberger, F. Xu, Overview of 3GPP LTE-advanced carrier aggregation for $4 \mathrm{G}$ wireless communications, IEEE Communications Magazine, (2012), Vol.50, No.2, pp.122-130, DOI: 10.1109/MCOM.2012.6146491

[11] Y. Wang, K. I. Pedersen, T. B. Sorensen, P. E. Mogensen, Carrier load balancing and packet scheduling for multi-carrier systems, IEEE Transactions on Wireless Communications, (2010), Vol.9, No.5, pp.1780-1789, DOI: 10.1109/TWC.2010.05.091310

[12] K. I. Pedersen, F. Frederiksen, C. Rosa, H. Nguyen, L. G. U. Garcia, Y. Wang, Carrier aggregation for LTE-advanced: functionality and performance aspects, IEEE Communications Magazine, (2011), Vol.49, No.6, pp.89-95, DOI: 10.1109/MCOM.2011.5783991

[13] P. Bhat, S. Nagata, L. Campoy, I. Berberana, T. Derham, G. Liu, X. Shen, P. Zong, J. Yang, LTE-advanced: and operator perspective, IEEE Communications Magazine, (2012), Vol.50, No.2, pp.104-114, DOI: 10.1109/MCOM.2012.6146489

[14] M. Cai, J. N. Laneman, B. Hochwald, Carrier aggregation for phase-array analog beamforming with beam squint, GLOBECOM 2017 - 2017 IEEE Global Communications Conference, (2017), December 4-8; Singapore.

[15] C. S. Park, L. Sundstrom, A. Wallen, A. Khayrallah, Carrier aggregation for LTE-advanced: design challenges of terminals, IEEE Communications Magazine, (2013), Vol.51, No.12, pp.76-84, DOI: 10.1109/MCOM.2013.6685761

[16] X. Gao, L. Dai, S. Han, C.-L. I, R. W. Heath, Energy-efficient hybrid analog and digital precoding for mmWave MIMO systems with large antenna arrays, IEEE Journal on Selected Areas in Communications, (2016), Vol.34, No.4, pp.998-1009, DOI: 10.1109/JSAC.2016.2549418

[17] D. R. Pehlke, K. Walsh, LTE-advanced pro RF front-end implementations to meet emerging carrier aggregation and DL MIMO requirements, IEEE Communications Magazine, (2017), Vol.55, No.4, pp.134-141, DOI: 10.1109/MCOM.2017.1601221 
[18] S. C. Hwu, B. Razavi, An RF receiver for intra-band carrier aggregation, IEEE Journal of Solid-State Circuits, (2015), Vol.50, No.4, pp.946-961, DOI: 10.1109/JSSC.2014.2386895

[19] N. Ishikawa, R. Rajashekar, S. Sugiura, L. Hanzo, Generalized-spatial-modulation-based reduced-RF-chain millimeter-wave communications, IEEE Transactions on Vehicular Technology, (2016), Vol.66, No.1, pp.879-883, DOI: $10.1109 /$ TVT.2016.2555378

[20] X. Gao, L. Dai, A. M. Sayeed, Low RF-complexity technologies to enable millimeter-wave MIMO with large antenna array for 5G wireless communications, IEEE Communications Magazine, (2018), Vol.56, No.4, pp.211-217, DOI: 10.1109/MCOM.2018.1600727

[21] T. E. Bogale, L. B. Le, A. Haghighat, L. Vandendorpe, On the number of RF chains and phase shifters, and scheduling design with hybrid analog-digital beamforming, IEEE Transactions on Wireless Communications, (2016), Vol.15, No.5, pp.3311-3326, DOI: 10.1109/TWC.2016.2519883

[22] S. Boyd, L. Vandenberghe, Convex optimization, Cambridge University press, (2004) 